\title{
Vinculación universidad-comunidad: pertinencia pedagógica- social de los programas nacionales de formación
}

\author{
Alexa Senior-Naveda ${ }^{1}$, Tibisay González², Freddy Marín-González ${ }^{\star}$, y Mercy Narváez Castro³, \\ (1) Departamento de Humanidades, Universidad de la Costa, Atlántico - Colombia. \\ (Correo-e: asenior@cuc.edu.co; fmarin1@cuc.edu.co) \\ (2) Programa de Ingeniería Civil, Universidad Nacional Experimental Politécnica “Alonso Gamero", Falcón - Venezuela. \\ (Correo-e: sociologagonzalez@gmail.com) \\ (3) Programa de Administración y Contaduría Pública, Núcleo Punto Fijo, Universidad del Zulia, Falcón- Venezuela. \\ (Correo-e: mercynarvaez@gmail.com)
}

* Autor a quien debe ser dirigida la correspondencia

Recibido Ene. 8, 2021; Aceptado Mar. 13, 2021; Versión final May. 11, 2021, Publicado Ago. 2021

\begin{abstract}
Resumen
Este estudio tiene por objetivo analizar la vinculación universidad-comunidad sobre la base de pertinencia pedagógica-social de los proyectos "unidades curriculares en los programas nacionales de formación" que se adelantan en el Instituto Universitario de Tecnología Alonso Gamero (Venezuela). El estudio se estructuró en una primera fase a través de la construcción de un sistema teórico, seguido de una fase de campo de naturaleza descriptiva en la cual se hizo uso de un cuestionario aplicado a una población de diez coordinadores departamentales pertenecientes a la institución. Los resultados indican que existe una significativa presencia de: productos generados para el desarrollo comunitario, articulación con la realidad social, transformación en el entorno y articulación teórico-práctica. Se concluye que la gestión efectiva de las unidades curriculares permite la articulación entre las funciones sustantivas de la universidad y la interacción socio comunitaria, ofreciendo a los estudiantes oportunidades para desarrollar y socializar sus competencias.
\end{abstract}

Palabras clave: vinculación universidad; comunidad; pertinencia pedagógica-social; programas; formación; unidades curriculares

\section{University-community link: socio-pedagogical relevance of national training programs}

\begin{abstract}
The present study aims to assess the link between university and community in the socio-pedagogical relevance of "the curricular units of the national training programs" project at the University Institute of Technology Alonso Gamero (Venezuela). The first phase consists on developing a theoretical system that is followed by a descriptive field phase in which a questionnaire was applied to a sample of ten faculty coordinators. The results show that there is a significant presence of: community development products, social reality articulation, surroundings transformation, and theoretical-practical articulation. It is concluded that effective management of curricular units allows coordinating university goals and socio-community interactions, opening opportunities for students to develop and socialize their skills.
\end{abstract}

Keywords: university-community link; substantive functions; training programs; curricular units 


\section{INTRODUCCIÓN}

La sociedad se encuentra inmersa en un proceso acelerado de cambio en todas las esferas de la vida humana. Las exigencias de transformaciones más profundas están en la organización y operación de la educación en general; muy especialmente, de la educación universitaria, la cual para el cumplimiento de su misión debe transformarse profundamente, haciéndose orgánicamente más flexible, diversificándose en sus estructuras, planes de estudios, mallas curriculares, formas de organización, entre otros, para de esta manera dar respuestas a las diferentes exigencias en los campos: científico, tecnológico, social y cultural. En consonancia con lo anterior, Alcántar y Arcos (2004), plantean que la transformación de las instituciones universitarias se debe enmarcar en sus formas de trabajo, las cuales resultan rígidas para los requerimientos que la sociedad impone pues, la velocidad de respuesta de las mismas en muchas ocasiones no satisface a un mercado que cada vez es más demandante. A esto se suma, la falta de comunicación e interacción entre las unidades académicas para trabajar en proyectos conjuntos, y la débil correlación de esfuerzos entre las funciones sustantivas de docencia, investigación y extensión de la cultura y los servicios, así como de la administración; no por la falta de voluntad, sino debido a inercias de trabajo y fines tradicionalmente separados unos de otros. (Estévez et al., 2015)

Ahora bien, dentro de las opciones que tienen las universidades para replantear la función que cumplen está el modelo de vinculación armónica con el entorno. De acuerdo con Novillo Díaz (2019) y Williamson et al., (2016) por medio de la vinculación es posible detectar problemas que luego serán tratados mediante estudios para ser corregidos o resueltos a través de la creación de programas con la comunidad (Polaino et al., 2017). Este proceso es viable si se logra la adaptación de las funciones sustantivas a los retos que impone la sociedad actual. En tal sentido, Jongbloed et al., (2008) indican que las universidades a escala global deben estar comprometidas a reconsiderar su papel y las relaciones con los diversos grupos, partes interesadas o comunidades con las que interactúan atendiéndolos según sus requerimientos y necesidades. De acuerdo a su importancia. De allí que las funciones sustantivas de docencia, investigación y extensión con la sociedad han de ser planeadas con miras a contribuir al desarrollo integral de la sociedad con la que interactúan (Rykun et al., 2015 y Gómez, 2020)

De acuerdo con Malagón-Plata (2006) el nuevo modelo educativo de vinculación tendrá cambios pedagógicos importantes donde el rol de profesor y estudiante serán modificados, redefinidos, haciéndose menos jerárquicos, poco dependientes y más colaborativos e interactuantes. El conocimiento será apropiado de diferentes maneras, dentro y fuera de la universidad. La creatividad, la imaginación, la reflexión y el trabajo colectivo constituyen estrategias para el trabajo cognoscitivo. El campus universitario no tendrá límites y el aprendizaje será durante toda la vida, sin restricciones de tiempo y lugar (Di Bello y Romero 2018; Cisneros et al, 2018). En adición a lo anterior, se puede afirmar que el trabajo de las universidades debe ir más allá de las funciones sustantivas de docencia, investigación y extensión, es decir, estas instituciones tienen también la misión social de generación y difusión del conocimiento logrando el establecimiento de una orientación bidireccional entre las mismas con su comunidad. En correspondencia con esta afirmación, De Aparicio et al., 2007 y Rueda et al., 2020, indican que la vida universitaria se desenvuelve bajo la égida de tres gestiones fundamentales: investigación, docencia y extensión por medio de la vinculación con la sociedad.

Lo anterior implica la decisión de la universidad en ponerse al servicio del medio social en el cual se inserta. De modo que la vinculación con la sociedad se constituye en la gestión principal que se erige sobre la investigación, la docencia y extensión, a tenor de dar respuesta a las demandas que la misma exige. De acuerdo con Gould Bei (2002), la vinculación es un proceso integral que atiende las funciones sustantivas de docencia, investigación y extensión de la cultura y los servicios de las instituciones de Educación Universitaria para su interacción eficaz y eficiente con el entorno socio económico mediante el desarrollo de acciones y proyectos de beneficio mutuo, que contribuyan a su posicionamiento y reconocimiento social.

Desde la perspectiva de Williamson et al., (2016) se espera entonces que las universidades contribuyan con la sociedad por medio de sus comunidades locales mediante algunas iniciativas de vinculación, tales como: incluir estudiantes universitarios que se ofrecen como voluntarios en las escuelas del vecindario, profesores que realizan actividades de investigación o líderes universitarios que promueven importantes iniciativas de desarrollo comunitario. A menudo, estas iniciativas dan como resultado nuevos conocimientos para la academia y experiencias transformadoras para los miembros de la comunidad (Grunwell y Ha, 2014).

Ahora bien, de acuerdo con Roth (2011) para alcanzar con éxito el anterior planteamiento, las universidades y sus comunidades anfitrionas deben formular estrategias de vinculación que resulten en logros mutuamente beneficiosos. Dentro de las estrategias que se proponen, Molina et al. (2015) sugieren la promoción de lazos de confianza entre la Institución de Educación Superior y la comunidad lo cual forja un compromiso institucional para resolver problemas que se presenten en la misma, ayudan a crear una cadena de valorización e incrementa el grado de satisfacción a través de una relación útil y solidaria (Rodelo y Castro, 2016; Kromydas, 2017). 
De igual manera se puede afirmar que en la formulación e implementación de estrategias de vinculación el liderazgo universitario juega un papel clave tanto en la determinación de la participación de la universidad en la comunidad como en la actitud de la comunidad hacia la universidad (Black, 2015; Marín et al., 2017; Avila et al.,2016; Veletic y Vegar, 2021). Cuando las universidades, los gobiernos locales y los líderes comunitarios desarrollan un enfoque estratégico de colaboración para el desarrollo económico, los resultados resultan positivos con beneficios para todas las partes involucradas (Grunwell y Ha, 2014; Padilla y Marúm, 2004). A la luz de los anteriores planteamientos se puede apuntar que las universidades deben establecer una relación más significativa con el entorno sustentándose en valores institucionales de colaboración, de tal manera, que se generen procesos sinérgicos respaldados en lineamientos estratégicos explícitos de actuación con la comunidad. Todo ello con el propósito de fomentar y asegurar ambientes propicios para el desarrollo de la sociedad y las mismas universidades. (Heng et al., 2020)

Así, para efectos de este trabajo se presenta el caso específico del Instituto Universitario de Tecnología "Alonso Gamero" (IUTAG), ubicado en la ciudad de Santa Ana de Coro del Estado Falcón - Venezuela. Dicha Institución, ofrece los llamados Programas Nacionales de Formación (PNF), los cuales tienen como propósito constituir redes de aprendizaje para la generación, transformación y apropiación social del conocimiento en determinadas áreas. En particular, los PNF buscan promover progresivamente la articulación y cooperación solidaria entre las Instituciones Universitarias, con los organismos del estado, empresas, organizaciones sociales y comunitarias (MPPPEU, 2009). EI IUTAG ofrece PNF en: Administración, Agroalimentaria, Informática, Contaduría Pública, Construcción Civil, Instrumentación, Electricidad y Controles, Procesos Químicos, atendiendo una población aproximadamente de siete mil cincuenta (7.050) estudiantes distribuidos de la siguiente manera: PNF: cuatro mil trecientos diez (4.310), Prosecución: dos mil trecientos cincuenta y uno (2351), Procedentes de todo el país y en la Municipalización en los ambientes académicos de Dabajuro, Federación (Churuguara) y Pedregal: trecientos ochenta y nueve (389) estudiantes.

Según lo instituido por Ministerio del Poder Popular para la Educación Universitaria (MPPPEU) en Gaceta Oficial de la República Bolivariana de Venezuela № 38.930, Resolución № 2.963, de fecha 14 de mayo del 2008, el IUTAG está autorizado para la gestión de los Programas Nacionales de Formación (PNF), a cargo de las instituciones de Educación Superior. Conforme a lo establecido en esta normativa, en la universidad se debe contribuir decididamente a la solución de los críticos problemas que aquejan a la sociedad, atendiendo a las necesidades que se evidencien, las cuales deben percibirse a través de los diagnósticos realizados en los abordajes e intervenciones a las comunidades, ya sean de orden social, económico o político. De allí que, es a través de la unidad curricular de "Proyecto" que la institución pone en marcha la nueva concepción de la educación universitaria, con una nueva filosofía (Williamson et al., 2016). Es importante mencionar que "Proyecto" se define por el MPPPEU (2009) como una unidad curricular de integración de saberes y contraste entre la teoría y práctica; se constituye en el eje central de los Programas Nacionales de Formación (PNF), su aplicabilidad radica en la resolución de problemas y el desarrollo de potencialidades. Los "Proyectos" están vinculados con los planes estratégicos del gobierno venezolano y están dirigidos a consolidar la soberanía política, tecnológica, económica, social y cultural.

Igualmente, la Misión Alma Mater del Ministerio del Poder Popular para la Educación Universitaria (MPPPEU, 2009), promueve un nuevo concepto de la Educación Universitaria que estimule los valores bolivarianos para alcanzar una sociedad más justa e inclusiva, impulsar la transformación, propulsar su articulación tanto territorial como con el Proyecto Nacional de Desarrollo; constituyéndose como referencia de una nueva institucionalidad caracterizada por la cooperación solidaria cuyo eje es la generación, transformación, socialización de conocimiento pertinente a las realidades y retos culturales, ambientales, políticos, económicos y sociales. Por otra parte, conviene destacar que a través de la Unidad Curricular de "Proyecto", en concordancia con el Proyecto Nacional Simón Bolívar: Líneas Generales del Plan de Desarrollo Económico y Social de la Nación (2007 - 2013) y el Plan de la Patria (2013-2019), se inserta a las Instituciones de Educación Universitaria con las comunidades con el propósito de que las mismas gestionen los principales problemas que se presentan (Peyton et al., 2019).

Ahora bien, en el marco de los lineamientos gubernamentales del estado venezolano previamente referidos, en el IUTAG se realizan prácticas que ayudan a generar equipos interdisciplinarios en los cuales intervienen y vinculan a la institución con los diferentes territorios a través de abordajes comunitarios, con el acompañamiento de enlaces territoriales e institucionales, estudiantes y los Consejos Comunales. Todo ello con el propósito de que se alcance la pertinencia pedagógica y social de la Universidad, reconociendo que se aprende a través del dialogo de saberes para contribuir con el desarrollo, se conjuga el conocimiento teórico con la práctica, preparando así un participante que sea capaz de enfrentar cualquier reto de la vida y comprometido con la realidad social. De acuerdo con Estupiñan et al., (2016) la pertinencia pedagógica y social de la Universidad se constituye en el proceso en el cual el universo cognitivo, académico e investigativo adquirido se complementa con la praxis social y desde ahí se articulan en función de respuestas a problemáticas comunes del contexto social. 
Por su parte, la United Nations Educational, Scientific and Cultural Organization (UNESCO) plantea que, la pertinencia pedagógica - social de la educación superior se corresponde con lo que la sociedad espera de las instituciones universitarias y lo que éstas hacen al respecto. "Para ello se requiere normas éticas, imparcialidad política, capacidad crítica y, al mismo tiempo, una mejor articulación con los problemas de la sociedad y del mundo del trabajo, fundando las orientaciones a largo plazo en objetivos y necesidades societales, comprendidos el respeto de las culturas y la protección del medio ambiente. Principios como la contextualización y pertinencia resultan factores clave para fortalecer la relación entre la universidad y el entorno, desde las funciones sustantivas de las instituciones de educación superior. Las dimensiones de gestión, curricular, didáctica y pedagógica, así como la responsabilidad social constituyen referentes para impulsar procesos de cooperación entre las organizaciones interactuantes" (UNESCO, 1998).

Ahora bien, lo pretendido es analizar la pertinencia pedagógica y social de los "Proyectos" que se adelantan en el IUTAG como forma de vinculación para generar y trasmitir conocimientos orientados a la comprensión y transformación de los contextos de acción de la sociedad a partir de las funciones sustantivas de la universidad. La trascendencia de este análisis se sustenta en el compromiso de desarrollo social que tiene la Universidad Venezolana de buscar que se generen espacios de reflexión e intercambio de saberes, enmarcados en un proceso educativo que permita la transformación socio productiva del país fomentando con ello la solución de las problemáticas de la comunidad.

\section{MATERIALES Y MÉTODOS}

Este estudio se enmarca en el "Enfoque Empírico- Analítico", también conocido como "Positivista", el cual se fundamenta en un estilo de pensamiento sensorial, en un lenguaje numérico-aritmético y en referencias de validación situadas en la "realidad objetiva". La ejecución de la investigación se estructuró siguiendo el sistema de variabilidad de la investigación educativa de Padrón (2019), con una perspectiva sistémica del proceso de investigación, en el cual en una primera fase se configura del sistema teórico deductivo, seguido de una segunda fase de campo de naturaleza descriptiva. En la primera fase lo que se pretende es establecer un sistema de relaciones teóricas que permitan fundamentar la Vinculación Universidad - Comunidad desde la pertinencia pedagógica y social. En esta fase se hizo uso del método lógico inferencial.

En lo referente a la fase de campo de esta investigación, se siguió un diseño de investigación de campo, no experimental aplicado a la población objeto de estudio de carácter finito, representada por diez (10) Coordinadores Departamentales de Proyecto por PNF del IUTAG, incluyendo al Coordinador de la Unidad Institucional de Proyecto (UNIIP-IUTAG). Para la recolección de información se diseñó como instrumento un cuestionario, el cual está estructurado bajo una escala tipo Likert de tres (03) alternativas de respuesta (Totalmente de Acuerdo, En Desacuerdo, Indiferente).

\section{RESULTADOS Y DISCUSIÓN}

A los fines de esta investigación la pertinencia pedagógica y social se analizó en concordancia con el nivel de correspondencia entre las necesidades y situaciones deficitarias de la comunidad (demandas) y la respuesta académica social de la universidad a partir de los "Proyectos" generados en el contexto de los PNF del IUTAG. En consecuencia, será dimensionada esta variable atendiendo a los siguientes aspectos: a) productos generados para el desarrollo comunitario, b) articulación con la realidad social, c) transformación y cambios en el entorno y d) articulación teórico-práctica.

Así, en relación con los productos generados para el desarrollo comunitario mediante la implementación de la unidad curricular "proyecto" se puede afirmar, según los datos observados en la Tabla 1, que para un significativo porcentaje de los encuestados (90\%) los saberes se integran en esta unidad curricular, lo cual permite la producción del conocimiento y brindar aportes a las comunidades. De la misma manera para el $80 \%$ de los Coordinadores los proyectos que ejecutan los estudiantes de los diferentes PNF desarrollan la producción de conocimiento y transferencia que se requieren por el sector social.

Igualmente, para el $70 \%$ de los Coordinadores Departamentales de Proyecto, entre las finalidades de la ejecución de esta unidad curricular está la generación de aplicaciones tecnológicas que mejoren procesos de la vida comunitaria, y además, a través de éstos los participantes transfieren tecnología a las comunidades, fortaleciendo la articulación de los nuevos saberes con los saberes ancestrales, tal como se observa en la Tabla 1. En lo correspondiente a la articulación de la unidad curricular "proyecto" con la realidad social comunitaria se puede indicar que para el $70 \%$ de los coordinadores consultados el conocimiento generado en los procesos investigativos es pertinente con las necesidades de los colectivos sociales y se encuentran disponibles, para ser empleados por las comunidades en el momento que sea requerido. 
Tabla 1: Productos generados para el desarrollo comunitario

\begin{tabular}{|l|c|c|c|c|c|c|c|c|}
\hline \multirow{2}{*}{ Productos generados para el desarrollo comunitario } & \multicolumn{2}{|c|}{$\begin{array}{l}\text { Totalmente } \\
\text { de acuerdo }\end{array}$} & \multicolumn{2}{|c|}{$\begin{array}{c}\text { En } \\
\text { desacuerdo }\end{array}$} & \multicolumn{2}{|c|}{ Indiferente } & \multicolumn{2}{|c|}{ Total } \\
\cline { 2 - 10 } & $F r$ & $\%$ & $F r$ & $\%$ & $F r$ & $\%$ & $F r$ & $\%$ \\
\hline $\begin{array}{l}\text { En la unidad curricular de Proyecto, se integran los saberes } \\
\text { generando la producción del conocimiento y brindando } \\
\text { aportes a las comunidades }\end{array}$ & 9 & 90 & 1 & 10 & - & - & 10 & 100 \\
\hline $\begin{array}{l}\text { Los proyectos que ejecutan los estudiantes de los diferentes } \\
\text { PNF desarrollan la producción de conocimiento y } \\
\text { transferencia requerido por el sector social }\end{array}$ & 8 & 80 & 1 & 10 & 1 & 10 & 10 & 100 \\
\hline $\begin{array}{l}\text { Los proyectos ejecutados en la Institución se realizan con la } \\
\text { finalidad de generar aplicaciones tecnológicas que mejoren } \\
\text { procesos de la vida comunitaria }\end{array}$ & 7 & 70 & 2 & 20 & 1 & 10 & 10 & 100 \\
\hline $\begin{array}{l}\text { Mediante los Proyectos, los participantes transfieren } \\
\text { tecnología a las comunidades, fortaleciendo la articulación de } \\
\text { los nuevos saberes con los saberes ancestrales }\end{array}$ & 7 & 70 & 3 & 30 & - & - & 10 & 100 \\
\hline
\end{tabular}

Del mismo modo, para la totalidad de los encuestados (100\%) a través de los Programas Nacionales de Formación (PNF) se vinculan los procesos formativos con el abordaje de los problemas en contextos reales. Destaca además, que los proyectos (como unidades curriculares) se constituyen en espacios de aprendizaje que posibilitan el abordaje de los problemas del entorno y sus posibles soluciones favorecen la transferencia de conocimiento hacia las comunidades, así se evidencia en la Tabla 2.

Tabla 2: Articulación con la realidad social

\begin{tabular}{|l|c|c|c|c|c|c|c|c|}
\hline \multirow{2}{*}{ Articulación con la realidad social } & \multicolumn{2}{|c|}{$\begin{array}{l}\text { Totalmente } \\
\text { de acuerdo }\end{array}$} & \multicolumn{2}{|c|}{$\begin{array}{c}\text { En } \\
\text { desacuerdo }\end{array}$} & \multicolumn{2}{l|}{ Indiferente } & \multicolumn{2}{|c|}{ Total } \\
\cline { 2 - 12 } & $F r$ & $\%$ & $F r$ & $\%$ & $F r$ & $\%$ & $F r$ & $\%$ \\
\hline $\begin{array}{l}\text { El conocimiento generado en los procesos investigativos es } \\
\text { pertinente con las necesidades de los colectivos sociales y } \\
\text { están disponibles, para ser empleados por las comunidades }\end{array}$ & 7 & 70 & 2 & 20 & 1 & 10 & 10 & 100 \\
\hline $\begin{array}{l}\text { Los PNF vinculan el ejercicio formativo, con el abordaje de los } \\
\text { problemas en contextos reales }\end{array}$ & 10 & 100 & - & - & - & - & 10 & 100 \\
\hline $\begin{array}{l}\text { Los proyectos constituyen espacios de aprendizaje que } \\
\text { posibilitan el abordaje de los problemas del entorno y sus } \\
\text { posibles soluciones favorecen la transferencia de conocimiento } \\
\text { hacia las comunidades }\end{array}$ & 10 & 100 & - & - & - & - & 10 & 100 \\
\hline
\end{tabular}

Ahora bien, en lo concerniente a la transformación y cambios en el entorno, los aspectos más destacados indican que el $80 \%$ de la plana directiva consultada manifiesta que en el contexto de los PNF la Universidad se concibe como un sistema abierto, cuyas acciones se orientan en función de las demandas sociales, facilitando de esta manera la integración con la comunidad. Por otra parte, para la mayoría de los consultados (70\%) la vinculación Universidad - Comunidad se constituye en un escenario propicio para incrementar el capital relacional a través de los "Proyectos" logrando alianzas y redes con entes gubernamentales, Comunidades, Consejos Comunales y otros Organismos. Del mismo modo, para el $80 \%$ del personal consultado, la vinculación Universidad - Comunidad posibilita el reforzamiento y la renovación del proceso de mediación docente, interconectando la visión del mundo, trabajo y sociedad con la perspectiva científica tecnológica, tal como se observa en la Tabla 3.

Coincidiendo con la información anteriormente descrita (Buys y Bursnall, 2007; Yaw-Akeparep, 2019) indican que son muchas las ventajas de la vinculación universidad - comunidad, destacan: mejores oportunidades para que los estudiantes puedan aprender a través de la experiencia laboral en proyectos comunitarios, diseño de nuevos programas académicos para satisfacer las necesidades en la comunidad, aumento de las oportunidades de trabajo para los graduados, mejora en los procesos de enseñanza aprendizaje, entre otros beneficios. En correspondencia con la información previamente expuesta en esta sección del artículo, Chatterton y Goddard (2000) indican que, las instituciones de educación superior deben responder al entorno cambiante, estableciendo novedosas estructuras de gestión institucional para satisfacer de manera más efectiva las demandas de los interesados regionales. Tales cambios están ocurriendo no solo en el contexto de los roles tradicionales de las universidades, sino además dentro de su rol de servicio a las comunidades (Huerta-Rivero et al., 2020). Igualmente, un desafío clave para las instituciones de educación superior es mejorar el papel que éstas juegan, a través de su personal y estudiantes, en la construcción y desarrollo de 
redes de confianza y compromiso cívico con diferentes relacionados, y por lo tanto, en el ámbito político comunitario y en el liderazgo cultural de sus localidades (Black, 2015; Marín et al., 2019; Velectic y Vegar 2021).

Tabla 3: Transformación y cambios en el entorno

\begin{tabular}{|c|c|c|c|c|c|c|c|c|}
\hline \multirow{2}{*}{ Transformación y cambios en el entorno } & \multicolumn{2}{|c|}{$\begin{array}{l}\text { Totalmente } \\
\text { de acuerdo }\end{array}$} & \multicolumn{2}{|c|}{$\begin{array}{c}\text { En } \\
\text { desacuerdo }\end{array}$} & \multicolumn{2}{|c|}{ Indiferente } & \multicolumn{2}{|c|}{ Total } \\
\hline & $\mathrm{Fr}$ & $\%$ & $\mathrm{Fr}$ & $\%$ & $\mathrm{Fr}$ & $\%$ & $\mathrm{Fr}$ & $\%$ \\
\hline $\begin{array}{l}\text { En el contexto de los PNF la Universidad se concibe como un } \\
\text { sistema abierto, cuyas acciones se orientan en función de las } \\
\text { demandas sociales, facilitando de esta manera la integración } \\
\text { con la comunidad. }\end{array}$ & 8 & 80 & 2 & 20 & - & - & 10 & 100 \\
\hline $\begin{array}{l}\text { La vinculación Universidad - comunidad es un escenario } \\
\text { propicio para incrementar el capital relacional a través de los } \\
\text { Proyectos logrando alianzas y redes con entes gubernamen- } \\
\text { tales, Comunidades, Consejos Comunales y otros Organismos }\end{array}$ & 7 & 70 & 3 & 30 & - & - & 10 & 100 \\
\hline $\begin{array}{l}\text { La vinculación Universidad - Comunidad posibilita tanto el } \\
\text { reforzamiento como la renovación del proceso de mediación } \\
\text { docente, interconectando la visión del mundo, trabajo y } \\
\text { sociedad con la perspectiva científica - tecnológica. }\end{array}$ & 8 & 80 & 2 & 20 & - & - & 10 & 100 \\
\hline
\end{tabular}

En referencia a la articulación teórico-práctica de los Proyectos, el $80 \%$ de los encuestados indican que los mismos son considerados como Unidades Curriculares que permiten la integración de saberes, en los cuales se contrasta el componente teórico con el metodológico, articulando a su vez la teoría con la práctica. En la misma proporción (80\%) los consultados manifiestan que en las Instituciones de Educación Universitaria que funcionan bajo los lineamientos de los PNF, se genera conocimiento científico que se evidencia en los proyectos que se realizan en la institución (Tabla 4).

Tabla 4: Articulación teórico - práctica

\begin{tabular}{|l|c|c|c|c|c|c|c|c|c|}
\hline \multirow{2}{*}{ Articulación teórico - practica } & \multicolumn{2}{|c|}{$\begin{array}{l}\text { Totalmente } \\
\text { de acuerdo }\end{array}$} & \multicolumn{2}{|c|}{$\begin{array}{c}\text { En } \\
\text { desacuerdo }\end{array}$} & \multicolumn{2}{|c|}{ Indiferente } & \multicolumn{2}{c|}{ Total } \\
\cline { 2 - 11 } & $F r$ & $\%$ & $F r$ & $\%$ & $F r$ & $\%$ & $F r$ & $\%$ \\
\hline $\begin{array}{l}\text { Los Proyectos son Unidades Curriculares de integración de } \\
\text { Saberes, en los cuales se contrasta el componente teórico con } \\
\text { el metodológico, articulando a su vez la teoría con la práctica. }\end{array}$ & 8 & 80 & 2 & 20 & - & - & 10 & 100 \\
\hline $\begin{array}{l}\text { En las Instituciones de Educación Universitaria que funcionan } \\
\text { bajo los lineamientos de los PNF, se genera conocimiento } \\
\text { científico que se evidencia en los Proyectos con la producción } \\
\text { de conocimiento }\end{array}$ & 8 & 80 & 2 & 20 & - & - & 10 & 100 \\
\hline
\end{tabular}

\section{Propuesta conceptual operativa para la vinculación universidad comunidad (U-C)}

La presentación de esta propuesta conceptual surge por la necesidad de configurar las dimensiones teóricas y el nivel operativo estratégico que servirán de base para la Vinculación Universidad - Comunidad en el Instituto Universitario de Tecnología Alonso Gamero. En tal sentido, se identifican cuáles serán los componentes que promoverán los espacios de reflexión y cooperación solidaria según lo establecido en los criterios fundamentales de los PNF. Del mismo modo, la propuesta conceptual - operativa plantea el diseño de lineamientos que viabilizan la vinculación universidad comunidad (U-C) con el propósito de establecer alianzas duraderas con las comunidades, desde la perspectiva de los Programas Nacionales de Formación. Esta vinculación U-C debe corresponder con el papel rector de las Universidades en los procesos de producción y transferencia del conocimiento. De la misma manera, se debe colocar al estudiante en primer plano en la perspectiva de una educación para toda la vida formando los profesionales que requiere el desarrollo social, económico, político, cultural de la humanidad. En tal sentido, se plantean dos dimensiones:

\section{Dimensión técnica-operativa}

Esta dimensión está relacionada con los lineamientos que establezcan alianzas verdaderas de vinculación universidad-comunidad, mediante la promoción de auténticos espacios de reflexión, garantizando el intercambio de saberes y experiencias, consolidando comunidades de conocimiento y aprendizaje dirigidas a la generación, transformación y apropiación social del conocimiento en beneficio de las comunidades que la integran, por lo tanto el contexto operativo estará representado por el IUTAG el cumplimiento de estas acciones facilitará la práctica de interacción comunidad-facilitador-estudiante. 


\section{Dimensión pedagógica-didáctica}

Esta dimensión se centra en la factibilidad en ofrecer lineamientos estratégicos, para que se dé la vinculación u-c, identificando los diferentes perfiles (estudiante, docente, institución y comunidad) en función de lograr tal vinculación. En tal sentido, se muestran los precitados perfiles (figura 1):

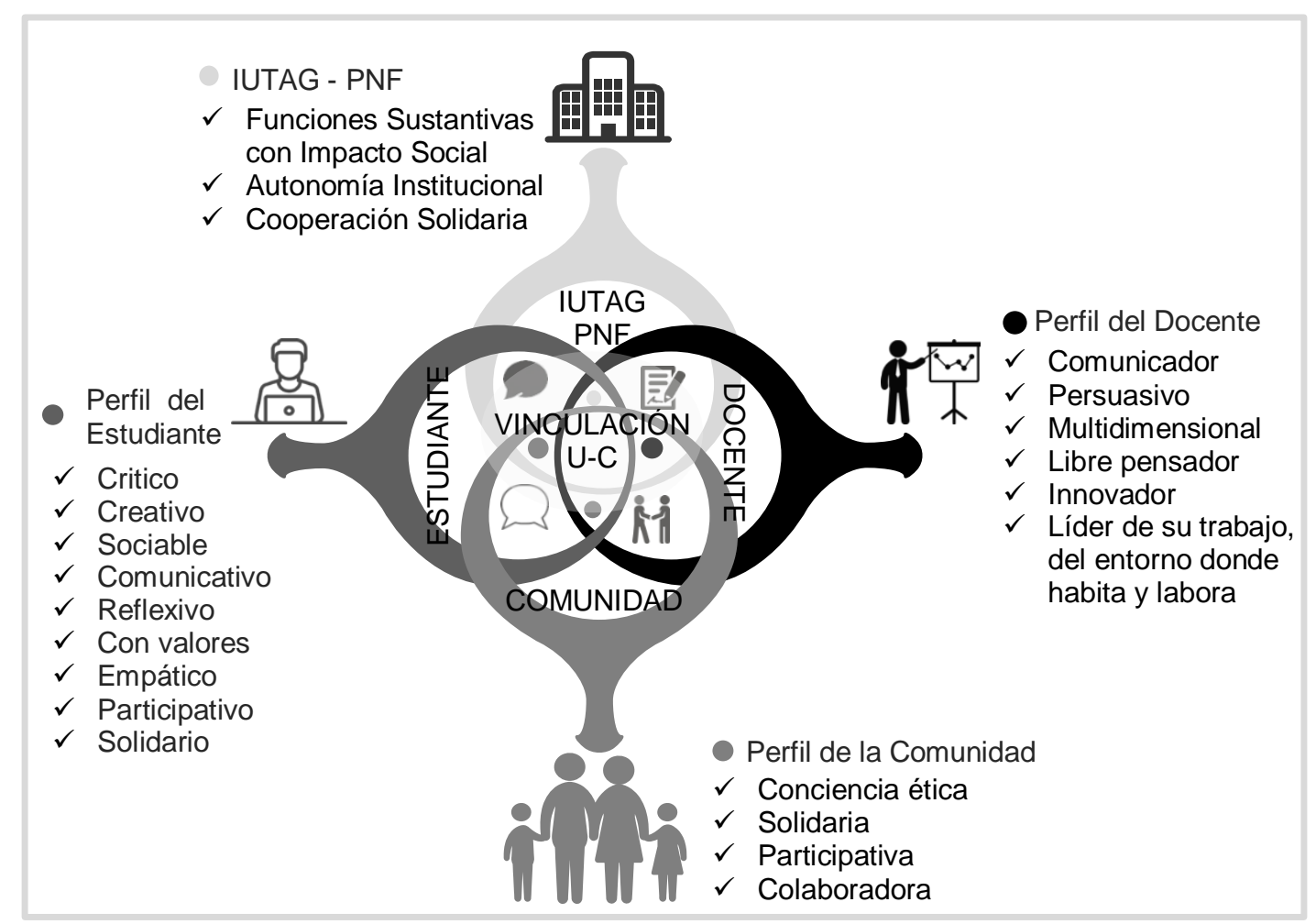

Fig.1: Perfiles de los Actores para la Vinculación U-C

Perfil del estudiante: se requiere de un estudiante proactivo, con plena participación, humanista, con sensibilidad social, crítica, inmersa en la nueva filosofía educativa, que se desenvuelva en equipos, capaz de adquirir conocimientos, pero también de aportarlos, comprometido con su entorno socio-ambiental, con valores y solidario, innovador y creativo. El estudiante que se desea tiene que ser: crítico, creativo, sociable, comunicativo, reflexivo, con valores, empático, participativo y solidario.

Perfil del docente: comunicador, persuasivo, multidimensional (profesional sensato, eficiente y líder de su gestión académica). Debe privilegiar el abordaje teórico del pensamiento sistémico y su praxis el trabajo en equipo. Libre pensador (autocontrolado, garante tanto de su propia calidad de vida académica como, la de sus estudiantes y el resto del entorno profesional), innovador y permanente reingeniador de sus conocimientos académicos profesionales, líder de su trabajo, del entorno donde habita y labora.

Perfil de la comunidad: los proyectos en el IUTAG se diseñan y ejecutan con la participación de los consejos comunales, respondiendo a las demandas locales, forjando herramientas de análisis, planificación que permitan hacer una evaluación permanente de las necesidades de formación y gestión. En tal sentido, se debe garantizar la articulación institucional para el desarrollo de programas de formación, creación intelectual y vinculación social compartidos, el intercambio de saberes y experiencias. La comunidad deberá poseer valores como: conciencia ética y solidaridad; además, debe ser participativa, colaboradora, responsable, comprometida e innovadora.

Perfil institucional: a través de la vinculación U-C, el IUTAG tendrá como principal propósito generar conocimientos dirigidos en primera instancia, a la solución de problemas "en, con y para" los contextos en los cuales la institución está inserta, y segundo, a la construcción de capacidades, coadyuvantes directamente con el desarrollo humano y sustentable. En correspondencia con este propósito, el perfil de la institución debe responder a las siguientes cualidades: autonomía institucional, desempeño de funciones sustantivas de docencia, investigación y vinculación con impacto social, cooperación solidaria con diferentes actores sociales. Lo descrito se evidencia en la Figura 1. 
Como evidencia de lo que se refleja en la investigación es importante reseñar que en el IUTAG se están realizando significativos esfuerzos por dar respuesta efectiva a las comunidades mediante la configuración de espacios de aprendizaje que posibilitan el abordaje de los problemas del entorno y la resolución de los mismos de manera conjunta (Universidad - Comunidad) lo cual propenderá al desarrollo comunitario sustentable del colectivo local en el cual está inserta la institución.

\section{CONCLUSIONES}

En atención al objetivo planteado en este artículo, se concluye que desde el punto de vista práctico, se visualiza la pertinencia social y pedagógica de los "Proyectos" que desde los PNF se desarrollan en el IUTAG. Además, la gestión efectiva de estas unidades curriculares permite la articulación entre las funciones sustantivas de la universidad y la interacción socio comunitaria. Por otra parte, ofrecen a los estudiantes oportunidades para desarrollar y socializar sus competencias. Del mismo modo, promueve el intercambio en diferentes contextos, la cooperación, la responsabilidad social institucional, el fomento de valores como: respeto, compromiso, solidaridad; y transforma profundamente la relación pedagógica profesores-estudiantes en la medida que se hace más personalizada y horizontal.

\section{REFERENCIAS}

Alcántar, V., y Arcos, J.L., La vinculación como instrumento de imagen y posicionamiento de las instituciones de educación superior, Revista electrónica de investigación educativa, 6(1), 01-12 (2004).

Ávila, L., Betancourt, A., Arias, G., y Ávila, A., Vinculación comunitaria y dialogo de saberes en la educación superior intercultural en México, Revista Mexicana de Investigación Educativa (RMIE), 21(70) (2016).

Black, S.A., Qualities of Effective Leadership in Higher Education, http://dx.doi.org/10.4236/ojl.2015.42006, Open Journal of Leadership, 4, 54-66 (2015).

Buys, N., y Bursnall S., Establishing university-community partnerships: Processes and benefits, https://doi.org/10.1080/13600800601175797, Journal of Higher Education Policy and Management, 29(1), 73-86 (2007).

Chatterton, P., y Goddard, J., The Response of Higher Education Institutions to Regional Needs, European Journal of Education, 35(4), 475-496 (2000).

Cisneros, P., y Mendoza, K., Vinculación universidad-sociedad: espacio para generar creatividad e innovación, https://doi.org/10.26871/killkana_social.v2i2.304, Revista Killkana Sociales, 2(2), 53-58 (2018).

De Aparicio, X., Chininin Macanchi, M.A., y Toledo Rodríguez, O., El rol de la vinculación en la integración de las funciones sustantivas de la Universidad Metropolitana del Ecuador, Revista Universidad y Sociedad, 9(4), 37-43 (2017).

Di Bello, M., y Romero, L., Vinculación y extensión universitaria: la relación entre la universidad y sus entornos en las universidades nacionales de Quilmes y Lanús, https://doi.org/10.21678/apuntes.82867, Apuntes, 82, 145-171 (2018).

Estévez, M., Maldonado, A., y Ramón, S., Una perspectiva de Evaluación de la calidad de la extensión universitaria, Universidad y Sociedad, 7(1E) (2015).

Estupiñan, J., Villamar, M., Campi, A., y Cadena, L., Reflexiones acerca de la pertinencia e impacto de la educación superior en Ecuador desde su perspectiva actual, Revista Órbita Pedagógica, 3(3), 81-92 (2016).

Gómez-Villamandos, J.C., El papel de la educación superior en el desarrollo sostenible, El Ágora diario del agua (2020).

Gould Bei, G., La administración de la vinculación ¿cómo hacer qué?, Tomo I, México (2002).

Grunwell, S., y Ha, I.S., How to revitalize a small rural town? An empirical study of factors for success: Universitycommunity collaboration with a small historic rural tourism town, Journal of Rural and Community Development, 9(2), 3250 (2014).

Heng, K., Hamid, M.O., y Khan, A., Factors influencing academics' research engagement and productivity: A developing countries perspective, Issues in Educational Research, 30(3), 965-987 (2020).

Huerta-Riveros, P., Gaete-Feres, H., y Pedraja-Rejas, L., Dirección estratégica, sistema de información y calidad. EI caso de una universidad estatal chilena, http://dx.doi.org/10.4067/S0718-07642020000200253, Inf. Tecnol, 31(2), 253266 (2020).

Jongbloed, B., Enders, J., y Salerno, C., Higher education and its communities: Interconnections, interdependencies and a research agenda, https://doi.org/10.1007/s10734-008-9128-2, Higher education, 56(3), 303-324 (2008).

Malagón Plata, L., La vinculación Universidad-Sociedad desde una perspectiva social, Educación y Educadores, 9(2), 79-93 (2006).

Marín-González, F. y otros cuatro autores, Gestión Participativa y Calidad Educativa en el contexto del Plan de Mejoramiento Institucional en Escuelas Colombianas, OPCIÓN, 33 (82), 344 - 365 (2017).

Marín-González, F., Talavera, R., Inciarte-González, A., y Avendaño-Villa, I., Redes de Cooperación CientíficoTecnológicas en Contextos Intersectoriales, http://dx.doi.org/10.4067/S0718-07642019000300013, Inf. Tecnol, 30(3), 1324 (2019). 
Molina, S., y otros cuatro autores, Prácticas sociales de personas mayores en el ámbito universitario: el voluntariado social como estrategia para la inclusión, Margen: Revista de trabajo social y ciencias sociales, 77 (2015).

MPPPEU: Ministerio del Poder Popular para la Educación Universitaria (Venezuela), Programas Nacionales de Formación en Educación Superior, Gaceta Oficial № 38.930, Resolución № 2.963, 14 de mayo, República Bolivariana de Venezuela (2009)

Novillo Díaz, L., La inclusión de grupos de atención prioritaria en proyectos de vinculación de la sociedad, Conrado: Revista pedagógica de la Universidad de Cienfuegos, 15(67), 75-80 (2019).

Padilla, J.T. y Marúm, E., La vinculación de la Universidad pública con el sector productivo, Revista de la Educación Superior, 129, 117-126 (2004).

Padrón, J., Fundamento teórico de las relaciones entre método, operaciones, técnicas e instrumentaciones, con referencia a la investigación científica, Nota 02 de ampliación para el seminario de Epistemología de la Investigación Cualitativa del Posdoctorado en Investigación Cualitativa del INICC, Lima (Perú) (2019).

Peyton, K., Sierra-Arévalo, M., y Rand, D.G., A field experiment on community policing and police legitimacy, https://doi.org/10.1073/pnas.1910157116 ,Proceedings of the National Academy of Sciences of the United States of America (PNAS), 116(40), 19894-19898 (2019).

Polaino, C.J., y Romillo, A., Vinculación con la sociedad en la Universidad de Otavalo, Ecuador, http://dx.doi.org/10.4067/S0718-50062017000300004, Formación Universitaria, 13(3), 21-30 (2017).

Rodelo-Sehuanes, M., y Castro-Bolaño, S., Reflexiones sobre la educabilidad: elemento fundamental en el proceso educacional del desarrollo humano, Cultura Educación y Sociedad, 7(2), 94-104 (2016).

Roth, K., Rural downtown revitalization strategies for university and community collaboration, SAM Advanced Management Journal, 76(3), 35-47 (2011).

Rueda, I., Acosta, B. y Cueva, F., Las universidades y sus prácticas de vinculación con la sociedad, https://doi.org/10.1590/ES.218154, Educ. Soc. Campinas, 41 (2020).

Rykun, A.Y., Yuzhaninov, K.M., y Vychuzhanina, E.V., Universities and Local Communities: Problems and Perspectives of Interaction, https://doi.org/10.1016/j.sbspro.2015.08.078, Procedia - Social and Behavioral Sciences, 200, $359-365$ (2015).

UNESCO: United Nations Educational, Scientific and Cultural Organization. World Declaration on Higher Education for the Twenty-first Century: Vision and Action, https://unesdoc.unesco.org/ark:/48223/pf0000113878 (1998)

Velectic, J., y Vegar, R., Developing a shared cluster construct of instructional leadership in TALIS, https://doi.org/10.1016/j.stueduc.2020.100942, Studies in Educational Evaluation,68 (2021).

Williamson, H.J., y otros seis autores, Community-University Partnerships for Research and Practice: Application of an Interactive and Contextual Model of Collaboration, J High Educ Outreach Engagem, 20(2), 55-84 (2016).

Yaw-Akparep, J., An Examination of the Causes of Students-Management Conflicts in University for Development Studies from 1999 to 2009, https://doi.org/10.4236/ojl.2019.82005, Open Journal of Leadership, 8, 75-94 (2019). 
